\title{
Predictive Factors for Neurological Deterioration after Surgical Decompression for Thoracic Ossified Yellow Ligament
}

\author{
Authors: $\quad{ }^{1}$ Chris Yuk Kwan Tang*, MBBS \\ ${ }^{1}$ Jason Pui Yin Cheung*, MBBS, MMedSc, FHKCOS, FHKAM, \\ FRCSEd \\ ${ }^{1}$ Dino Samartzis, DSc \\ ${ }^{1}$ Ka Hei Leung, MBBS, FHKCOS, FHKAM, FRCSEd \\ ${ }^{1}$ Yat Wa Wong, MBBS, FHKCOS, FHKAM, FRCSEd \\ ${ }^{1}$ Keith Dip Kei Luk, MBBS, MCh(orth), FRCS(Edin), \\ FRCS(Glas), FRACS, FHKCOS, FHKAM (ortho) \\ ${ }^{\mathbf{1}}$ Kenneth Man Chee Cheung, MBBS, MD, FRCS, FHKCOS, \\ FHKAM \\ *Dual first author \\ Affiliations: $\quad{ }^{1}$ Department of Orthopaedics and Traumatology, The University \\ of Hong Kong, Pokfulam, Hong Kong, SAR, China \\ Correspondence Kenneth Man Chee Cheung \\ Jessie Ho Professor in Spine Surgery \\ Head of Department \\ Professorial Block, 5th Floor, 102 Pokfulam Road \\ Department of Orthopaedics \& Traumatology \\ The University of Hong Kong \\ Queen Mary Hospital, Pokfulam Road \\ Hong Kong SAR, China \\ Tel: (+852) 2255-4341 \\ Fax: (+852) 2817-4392 \\ Email: cheungmc@hku.hk
}




\begin{abstract}
Purpose

Ossified yellow ligament (OYL) is one of the commonest causes of thoracic myelopathy. Surgical decompression is the mainstay treatment of myelopathy caused by OYL. However, factors related to post-operative neurological deterioration is unknown. Hence, the aim of study is to investigate the rate or predictive factors of post-operative neurological deterioration in OYL surgery.
\end{abstract}

\title{
Methods
}

A retrospective review was conducted for all southern Chinese patients with surgically treated thoracic OYL from January 1998 to December 2012. Clinical parameters under study included clinical presentation, distribution of OYL, preoperative walking assessment, pre- and postoperative neurological status, status of intraoperative neurophysiological monitoring, modified Japanese Orthopaedic Association (mJOA) score and complications. All outcomes were measured at postoperative 1 week and at 2 years.

\section{Results}

A total of 26 patients were included in this study. Most patients had Frankel grade D preoperatively. The rate of neurological deterioration was $15.4 \%$ and was correlated with presence of dural tear, extra-dural hematoma and spinal cord injury. Pre-operative walking score system was prognostic of patients' walking ability in the post-operative period. Intraoperative monitoring of Somatosensory Evoked Potentials (SSEP) was found to be useful in spinal cord injury monitoring in OYL operation, with a positive predictive value of $100 \%$ and negative predictive value of $92.3 \%$. The false negative rate of a SSEP signal drop was only $7.7 \%$

\section{Conclusions}

This is the first study exploring risk factors for post-operative neurological deterioration after surgery for thoracic OYL. The rate of neurological deficit is not small and prognostic factors for poor outcome include poor pre-operative walking score and intra-operative drop of SSEP signal.

\section{Introduction}


Ossified yellow ligament (OYL), also known as ossified ligamentum flavum $(\mathrm{OLF})$, is a common cause of thoracic myelopathy ${ }^{1-4}$. Aizawa et $a l^{3}$ reported that up to $75 \%$ of thoracic myelopathies are caused by OYL. The development of OYL is a result of endochondral ossification of the ligamentum flavum ${ }^{5}$ (Figure 1), which causes subsequent compressive myelopathy.

Many possible factors have been described in the literature as the cause of endochondral ossification in OYL such as mechanical stress ${ }^{5}$, overproduction of bone morphogenetic proteins (BMPs) 2, 4 \& 7 and transforming growth factor-ß (TGF-ß) ${ }^{6}$. Patients with OYL also have a higher prevalence of obesity, diabetes mellitus, hyperinsulinism and impaired glucose tolerance. ${ }^{7}$

This condition appears to be more common in Asian populations as evidenced by the majority of OYL studies in the region. ${ }^{3,-13}$ In one population-based study, the overall prevalence was reported to be $3.8 \%$ within a southern Chinese population. ${ }^{11}$ Yet, despite these epidemiological studies, there is limited clinical information regarding postoperative outcomes and complications ${ }^{2,3,8}$. Despite not discussed in the literature, many surgical complications are well-known to surgeons with regards to thoracic OYL surgery, including epidural hematoma, wound infection, dural tear and spinal cord injury. Dural ossification increases the chance of dural tear during laminectomy ${ }^{14}$. In a series of 110 patients undergoing multilevel laminectomies with no posterolateral fusion by Epstein, ${ }^{15} 10$ patients developed dural tears and all 10 (100\%) had corresponding levels of OYL. Three patients (30\%) had ossification extending to and through the dura in the lumbar levels. Thus OYL operations are high risk for dural tear, especially with dural adhesions. ${ }^{15}$ A study by Kawaguchi et $a l^{13}$ examined the variables affecting postsurgical prognosis for thoracic OYL in Japanese myelopethic patients. However, the neurological outcome was based on a Japanese Orthopaedic Association (JOA) score without further analyzing the specific subtype of neurological outcome, such as motor or sensory improvement and walking ability.

Due to limitations of previous studies, the rate of neurological deterioration after OYL surgery and the predictive factors associated with this poor outcome is still unknown. Thus, the aim of this study was to identify the prevalence of postoperative neurological deterioration and to identify predictive factors for neurological outcome in patients undergoing surgical treatment for thoracic OYL.

\section{??}

A study by Kawaguchi et $a l^{13}$ examined the variables affecting postsurgical prognosis for thoracic OYL in a Japanese population. However, the neurological outcome was only studied as a collective Japanese Orthopaedic Association (JOA) score without further analyzing the specific subtype of neurological outcome, such as motor or 
sensory improvement and walking ability.

Overall, there is only limited information for neurologic outcome, and it is the authors' impression that patients with thoracic myelopathy secondary to OYL are at particular high risk for neurologic deterioration after surgery. Thus the aim of the current study is to evaluate a single center's experience in the management of this specific condition with specific reference for postoperative neurologic outcome and factors that associated with such outcome.

\section{Materials and Methods}

A retrospective review was conducted, assessing myelopathic patients with surgically treated thoracic OYL from January 1998 to December 2012 in a single center. This study was approved by the local ethics review board. Patients with spine pathologies other than the thoracic region were excluded from the study. Cases with infection, tumors and previous reoperations were also excluded.

\section{Initial Presentation}

Clinical parameters under study included patient age, gender, mean time of presentation of symptoms to operation, presented symptoms and neurological status by Frankel grading ${ }^{16}$ (Table 1) at presentation were collected.

\section{Distribution of OYL}

The extensiveness of OYL over the spine was assessed by pre-operative CT scan and MRI. Levels and number of levels affected by OYL were collected.

\section{Neurological Status}

The walking ability is often affected in OYL patients and has significant impact on patient's activities of daily living. Objective criteria are needed to quantify the walking disability, hence the authors adopted a simple walking score grading scale. The pre-operative walking ability was stratified by the pre-operative walking score: score 0: cannot walk; score 1: unstable walking requiring aids for support; score 2: walking unaided with subjective instability; score 3: normal.

Frankel grade ${ }^{16}$ was used for assessment.

\section{Neurophysiological monitoring}

Intraoperative use of somatosensory evoked potentials (SSEP) for neurological monitoring was performed by neurophysiologist with at least 5 years experience. 
Motor evoked potential (MEP) monitoring was performed for 2 patients only. Any amplitude drop in signal was recorded. Significant drop was defined as more than $50 \%$ of drop from baseline. A 2x2 table was used to calculate the sensitivity, specificity, positive predictive value and negative predictive value. Sensitivity (true positive rate) was defined as those patients who had significant intra-operative drop in signal by SSEP and had neurological deterioration in the immediate post-operative period. Specificity (true negative rate) was defined as those who had no change in SSEP intra-operatively and did not have any neurological deterioration. Positive predictive value was calculated as the proportion of people with significant intra-operative signal drop who have spinal cord injury. Negative predictive value was calculated as the proportion of people without significant intra-operative signal drop who did not have spinal cord injury.

\section{Outcome measures}

Outcome measures included immediate (within 1 week post-operatively) and 2 years post-operative neurological recovery. These included lower limb motor power, sensation, sphincter function and walking abilities, modified JOA score, change of modified JOA score, presence of motor or sensory deficit and presence of post-operative complications.

Motor improvement was defined as muscle power improvement of at least 1 grade in at least 1 myotome of lower limbs by the MRC (Medical Research Council) grading. ${ }^{17}$ Sensory improvement was defined as decrease in the area of numbness or decreased in severity of numbness within the same area of involvement. Neurological improvement was defined as either motor or sensory improvement. Complications were also recorded.

\section{Statistical analysis}

Descriptive data was presented by means, ranges and standard deviation (SD). Statistical analysis was performed with Chi-squared test for discrete variables and analysis of variance (ANOVA) for multiple continuous variables. Fisher's exact test was used if the variable numbers were less than five. A p-value of $<0.05$ was considered significant.

\section{Results}

A total of 26 patients (16 males and 10 females) were included in the study. The mean age was 58 years old (range: 38 to 84 years, SD: 12.4 years). The mean time of symptom presentation to operation was 9.3 months (range from 0.5 to 48 months, SD: 13.0 months). All patients underwent decompression surgery and instrumented fusion 
was performed if intraoperative destabilization was evidenced after decompression or if deformity correction was required.

\section{Initial Presentation}

All patients presented with bilateral lower limb numbness. Seventeen patients (65.4\%) had bilateral lower limb weakness and 1 patient (3.8\%) had sphincter disturbance at initial presentation. Majority (92.3\%) of patients were in Frankel grade $\mathrm{D}$ at presentation.

\section{Distribution of OYL}

There were 7 segments (21.2\%) of OYL found in the upper thoracic spine (T1-4), 6 segments (18.2\%) in middle thoracic spine (T5-8) and 20 segments (60.6\%) in lower thoracic spine (T9-12). A total of 12 patients (46.2\%) had single level disease, 7 patients (26.9\%) had OYL affecting 2 levels and 7 patients (26.9\%) had OYL affecting 3 levels or more.

\section{Pre-operative Walking Score}

Regarding the pre-operative walking score, 7 patients (26.9\%) had score 0, 12 patients (46.2\%) had score 1 and 7 patients (26.9\%) had score 2.

\section{Post-operative Neurological Status}

Majority of patients had motor, sensory and neurological improvements in the immediate (within 1 week post-operatively) post-operative period, and further improved in the 2-year post-operative period. Similar improvements were seen for sphincter function and walking abilities. (Table 2)

Middle-thoracic OYL had the least improvement in the mean post-operative mJOA score, followed by upper-thoracic OYL. Lower-thoracic OYL had the largest improvement in the mJOA score. (Table 3) Multiple levels of OYL had the least improvement in mJOA score, followed by single level and 2-level involvement. A higher pre-operative walking score predicted a higher walking score at 2-year post-operative period ( $\mathrm{p}=0.002)$.

A higher pre-operative mJOA score was associated with a higher post-operative mJOA score $(p<0.05)$ ??check if very small, writes $<0.01$. The neurological outcome after operation was not associated with age and duration of symptoms in our series.

\section{Complications}

Five patients (19.2\%) had post-operative complications, which included two dural tears, one wound infection, one extra-dural hematoma and one spinal cord injury. 
Three (dural tear, extra-dural hematoma and spinal cord injury) of these 5 patients (60\%) had immediate post-operative neurological deterioration. All of them had residual symptoms at postoperative 2 years ( 2 had residual motor deficit, 4 had residual sensory deficit). All of them were in Frankel grade D at 2-year follow up. No statistical significant relationship could be drawn between the presence of complications and residual neurological symptoms at 2-year follow-up. ?p value

\section{Risk Factors for Post-operative Neurological Deterioration}

Four patients (15.4\%) had immediate neurological deterioration (Figure 2) postoperatively. Neurological deterioration was associated with the presence of post-operative complications, namely dural tear, extra-dural hematoma and spinal cord injury $(\mathrm{p}=0.01)$. All of the patients who experienced immediate neurological deterioration had residual symptoms at postoperative 2 years (50\% patients had less severe than pre-operative status, but both of them were still in Frankel grade D).

Risk of immediate neurological deterioration was not correlated with the type of OYL.

\section{Neurophysiological monitoring}

The role of intra-operative neurophysiological monitoring was also investigated. SSEP monitoring was performed for 22 patients (Table 4). Motor evoked potential (MEP) monitoring was performed for 2 patients only. One patient who had no change in MEP intra-operatively did not have any neurological deterioration (true negative). The other patient had complete disappearance of both SSEP and MEP intra-operatively and had neurological deterioration in the immediate post-operative period (true positive).

The absence of any significant intraoperative drop of SSEP predicted 2-year sensory improvement $(\mathrm{p}=0.048)$. The false negative rate of a drop in SSEP signal was 7.7\%. Failure to detect any signal preoperatively was not associated with poor neurological outcome. In our series, SSEP monitoring for spinal cord injury in OYL operation had a sensitivity of $50 \%$, specificity of $100 \%$, positive predictive value of $100 \%$ and negative predictive value of $92.3 \%$.

\section{Discussion}

This is the first study to analyze the incidence and possible risk factors of post-operative neurological deterioration and complications after surgical decompression for thoracic OYL. Our study found that OYL neurological 
deterioration was significantly associated with the presence of complications. Pre-operative walking score showed a good prediction of post-operative functional outcome. Intra-operative SSEP monitoring was a useful tool to monitor spinal cord injury in OYL operation.

In our series, all patients have bilateral lower limb numbness on presentation. Consistent with Guo et $\mathrm{al}^{11}$, our study showed that the lower thoracic spine (T9-12) was most commonly affected (60.6\%). This may be due to less anatomic protection from the rib cage, and thus more prone to degeneration because of high tensile forces present in the posterior column. Further study should be carried out to verify this.

The rate of neurological deterioration was $15.4 \%$ and was correlated with presence of complications, namely dural tear, extra-dural hematoma and spinal cord injury. A multivariate analysis for all spine surgery in $2012^{18}$ showed revision surgery, advanced age, and degenerative disease were significant risk factors for unintended dural tears. Surgeons should be more alert during surgery to dural tear when the above risk factors are present. Dural adhesions in OYL are common and thus, the decompression should be performed cautiously. As dural ossification increased the risk of dural tear in laminectomy, ${ }^{15}$ pre-operative identification of this is very important. In the bone window of axial CT scan, 2 radiological hallmarks of dural ossification in OYL were described by Muthukumar' ${ }^{19}$. The 'comma sign' was characterized by ossification of one-half of the circumference of the dura. The 'tram track sign' consisted of a hyperdense bony excrescence with a hypodense centre. Two methods were commonly employed intra-operatively to decrease the chance of dural tear in dural ossification. In the first method (slitting the dura), the dura mater was incised using a knife blade and nerve stripper to visualize the spinal cord. The void between the ossification and the spinal cord was utilized to excise the entire ossification. Small dural defects were directly sutured while large defects required patch repair. In the second method (floating the dura), the entire ossification was thinned as much as possible using a high-speed burr. The dura was dissociated from the ossification to cause it to bulge naturally. $\operatorname{Sun}^{20}$ compared 33 patients who underwent operation with either one method and found no significant differences between these 2 methods. The risk of dural tear should be decreased with the above methods and the risk could be predicted in pre-operative imaging.

The neurological deterioration was not predicted by age, duration of symptoms and pre-operative mJOA score. Regarding the surgical outcomes from previous studies, patients with lower pre-operative mJOA have a lower post-operative mJOA score $^{8}$, which is consistent with our study.

In our series, the pre-operative walking score described by us shows a prognostic value in determining future walking status. Spastic gait is the most disturbing 
symptom affecting a patient's daily function. The walking score developed by the authors can reflect the patient's functional activities and as it is simple to use, the authors suggest routine assessment using this walking score. Since a higher pre-operative walking score predicts higher post-operative walking score, earlier operation might be able to gain a more satisfactory outcome. However, this concept can only be validated with prospective studies.

Our study highlights specifically the use of SSEP as intra-operative neurophysiological monitoring for neurological deterioration. False negative rates are also low. In our series, only one patient, who had no significant intra-operative drop of SSEP signal, had neurological deterioration postoperatively, giving a false negative rate of 7.7\% (1 out of 13). In OYL surgery, SSEP for intra-operative spinal cord injury monitoring has a sensitivity of $50 \%$, specificity of $100 \%$, positive predictive value of $100 \%$ and negative predictive value of $92.3 \%$. Previous studies of intra-operative SSEP monitoring proposed sensitivity ranging from $0 \%^{21}$ to $100 \%^{22-24}$ and specificity ranging from $27 \%{ }^{25}$ to $100 \%{ }^{9}$ for detecting intra-operative spinal cord injury. The positive predictive value ranged from $15 \%{ }^{26}$ to $100 \% .{ }^{9}$ and the negative predictive value was $100 \%$ in one study. ${ }^{24}$ One previous study suggested a false-negative rate of $9 \%$ in SSEP monitoring which was similar to our results. ${ }^{21}$ These studies however included all spinal operations as a whole and none of them assessed the usage of SSPE specifically in OYL operation. A relatively low sensitivity with a high specificity of SSEP should alert the surgeons to identify the spinal cord injury whenever there is a significant drop in SSEP during OYL operation. The false negative rate for SSEP monitoring in OYL operation is relatively low. Thus, our findings suggest that SSEP is useful in monitoring in OYL surgery and the false negative rate is no worse than all spinal operations.

Nevertheless, there are some limitations to this study including its small sample size and retrospective design. Due to its retrospective nature, some of the important pre-operative parameters such as pre-operative proprioception, ankle clonus is not retrievable. Previous studies suggest that big toe proprioception impairment ${ }^{13}$ and ankle clonus ${ }^{27}$ are associated with poorer surgical outcome and these are parameters not analyzed in our study. In addition, some of the imaging data were not retrievable such as axial MRI scans. Furthermore, only drop in signal amplitude regarding intra-operative SSEP monitoring were recorded although increase in latency was also an important parameter. We could not draw any conclusion regarding the role of MEP in OYL operation in addition to SSEP. Only 2 subjects received intra-operative MEP monitoring. One patient had both SSEP and MEP monitoring intra-operatively. There was no signal drop and there was no neurological deterioration post-operatively. The other patient had complete disappearance of both SSEP and MEP intra-operatively 
and had neurological deterioration in the immediate post-operative period. A recent multicentre study ${ }^{28}$ showed that the sensitivity, specificity and false positive rates for MEP alone are $82 \%, 94 \%$ and $6.4 \%$ respectively. The authors in that study suggested that the most accurate modality combination is MEP and Br-SCEP (cord evoked potential after stimulation to the brain) with sensitivity of $90 \%$, specificity $94 \%$ and false positive rate of $6.1 \%$. Furthermore, the predictive factors proposed require further validation in a prospective manner.

\section{Conclusions}

This study specifically addresses the issue of neurologic outcome after OYL surgery in a southern Chinese population. We found that the complication rate for OYL operation was not low and post-operative neurological outcome in the presence of non-neurological complications was poor. The study noted that pre-operative walking score, intra-operative dural tear, haematoma formation and drop in SSEP signal are potential predictive risk factors that warrant future attention. This is also the first series to investigate the accuracy of SSEP to detect intra-operative spinal cord injury for OYL surgery. All patients are suggested to have intraoperative SSEP monitoring despite the small false negative rate. 


\section{References}

1 S. Mohindra, R. Chhabra, K. K. Mukherjee, S. K. Gupta, K. Vaiphei, V. K. Khosla, Spinal compression due to ossified yellow ligament: a short series of 5 patients and literature review, Surg Neurol 65(4): 377-84, discussion 384, 2006.

2 S. Mohindra, R. Gupta, R. Chhabra, S. K. Gupta, Compressive myelopathy due to ossified yellow ligament among South Asians: analysis of surgical outcome, Acta Neurochir (Wien) 153(3): 581-7, 2011.

3 T. Aizawa, T. Sato, H. Sasaki, F. Matsumoto, N. Morozumi, T. Kusakabe, E. Itoi, S. Kokubun, Results of surgical treatment for thoracic myelopathy: minimum 2-year follow-up study in 132 patients, J Neurosurg Spine 7(1): 13-20, 2007.

4 M. Payer, E. Bruder, J. A. Fischer, A. Benini, Thoracic myelopathy due to enlarged ossified yellow ligaments. Case report and review of the literature, J Neurosurg 92(1 Suppl): 105-8, 2000.

5 Munehito Yoshida, Pathology of Ossification of the Ligamentum Flavum, OPLL: 49-57, 2006.

6 K. Hayashi, Y. Ishidou, K. Yonemori, T. Nagamine, N. Origuchi, S. Maeda, T. Imamura, M. Kato, H. Yoshida, T. K. Sampath, P. ten Dijke, T. Sakou, Expression and localization of bone morphogenetic proteins (BMPs) and BMP receptors in ossification of the ligamentum flavum, Bone 21(1): 23-30, 1997.

7 Yasuhiro Takeuchi, Toshio Matsumoto, Yoh Takuwa, Yuichi Hoshino, Takahide Kurokawa, Naohiko Shibuya, Etsuro Ogata, High incidence of obesity and elevated serum immunoreactive insulin level in patients with paravertebral ligamentous ossification: A relationship to the development of ectopic ossification, J Bone Miner Metab 7: 17-21, 1989.

8 T. Aizawa, T. Sato, H. Sasaki, T. Kusakabe, N. Morozumi, S. Kokubun, Thoracic myelopathy caused by ossification of the ligamentum flavum: clinical features and surgical results in the Japanese population, J Neurosurg Spine 5(6): 514-9, 2006.

9 M. H. Khan, P. N. Smith, J. R. Balzer, D. Crammond, W. C. Welch, P. Gerszten, R. J. Sclabassi, J. D. Kang, W. F. Donaldson, Intraoperative somatosensory evoked potential monitoring during cervical spine corpectomy surgery: experience with 508 cases, Spine (Phila Pa 1976) 31(4): E105-13, 2006.

10 K. C. Kang, C. S. Lee, S. K. Shin, S. J. Park, C. H. Chung, S. S. Chung, Ossification of the ligamentum flavum of the thoracic spine in the Korean population, J Neurosurg Spine 14(4): 513-9, 2011.

11 J. J. Guo, K. D. Luk, J. Karppinen, H. Yang, K. M. Cheung, Prevalence, distribution, and morphology of ossification of the ligamentum flavum: a population study of one thousand seven hundred thirty-six magnetic resonance imaging scans, Spine (Phila Pa 1976) 35(1): 51-6, 2010.

12 N. Miyakoshi, Y. Shimada, T. Suzuki, M. Hongo, Y. Kasukawa, K. Okada, E. Itoi, Factors related to long-term outcome after decompressive surgery for ossification of the ligamentum flavum of the thoracic spine, J Neurosurg 99(3 Suppl): 251-6, 2003.

13 Y. Kawaguchi, T. Yasuda, S. Seki, M. Nakano, M. Kanamori, S. Sumi, T. Kimura, Variables 
affecting postsurgical prognosis of thoracic myelopathy caused by ossification of the ligamentum flavum, Spine J 13(9): 1095-107, 2013.

14 X. Sun, C. Sun, X. Liu, Z. Liu, Q. Qi, Z. Guo, H. Leng, Z. Chen, The frequency and treatment of dural tears and cerebrospinal fluid leakage in 266 patients with thoracic myelopathy caused by ossification of the ligamentum flavum, Spine (Phila Pa 1976) 37(12): E702-7, 2012.

15 N. E. Epstein, The frequency and etiology of intraoperative dural tears in 110 predominantly geriatric patients undergoing multilevel laminectomy with noninstrumented fusions, J Spinal Disord Tech 20(5): 380-6, 2007.

16 H. L. Frankel, D. O. Hancock, G. Hyslop, J. Melzak, L. S. Michaelis, G. H. Ungar, J. D. Vernon, J. $\mathrm{J}$. Walsh, The value of postural reduction in the initial management of closed injuries of the spine with paraplegia and tetraplegia. I, Paraplegia 7(3): 179-92, 1969.

17 Medical Research Council, Aids to examination of the peripheral nervous system, Memorandum no. 45. London: Her Majesty's Stationary Office 1976.

18 G. A. Baker, A. M. Cizik, R. J. Bransford, C. Bellabarba, M. A. Konodi, J. R. Chapman, M. J. Lee, Risk factors for unintended durotomy during spine surgery: a multivariate analysis, Spine $J$ 12(2): 121-6, 2012.

19 N. Muthukumar, Dural ossification in ossification of the ligamentum flavum: a preliminary report, Spine (Phila Pa 1976) 34(24): 2654-61, 2009.

20 J. Sun, C. Zhang, G. Ning, Y. Li, Y. Li, P. Wang, S. Feng, Surgical strategies for ossified ligamentum flavum associated with dural ossification in thoracic spinal stenosis, $J$ Clin Neurosci 21(12): 2102-6, 2014.

21 H. Deutsch, M. Arginteanu, K. Manhart, N. Perin, M. Camins, F. Moore, A. A. Steinberger, D. J. Weisz, Somatosensory evoked potential monitoring in anterior thoracic vertebrectomy, J Neurosurg 92(2 Suppl): 155-61, 2000.

22 S. L. Papastefanou, L. M. Henderson, N. J. Smith, A. Hamilton, J. K. Webb, Surface electrode somatosensory-evoked potentials in spinal surgery: implications for indications and practice, Spine (Phila Pa 1976) 25(19): 2467-72, 2000.

23 M. T. Stechison, S. G. Panagis, S. S. Reinhart, Somatosensory evoked potential. Monitoring during spinal surgery, Acta Neurochir (Wien) 135(1-2): 56-61, 1995.

24 Y. L. Leung, M. Grevitt, L. Henderson, J. Smith, Cord monitoring changes and segmental vessel ligation in the "at risk" cord during anterior spinal deformity surgery, Spine (Phila Pa 1976) 30(16): 1870-4, 2005.

25 D. M. May, S. J. Jones, H. A. Crockard, Somatosensory evoked potential monitoring in cervical surgery: identification of pre- and intraoperative risk factors associated with neurological deterioration, J Neurosurg 85(4): 566-73, 1996.

26 P. H. Manninen, Monitoring evoked potentials during spinal surgery in one institution, Can $J$ Anaesth 45(5 Pt 1): 460-5, 1998.

27 S. He, N. Hussain, S. Li, T. Hou, Clinical and prognostic analysis of ossified ligamentum flavum in 
a Chinese population, J Neurosurg Spine 3(5): 348-54, 2005.

28 Z. Ito, Y. Matsuyama, M. Ando, S. Kawabata, T. Kanchiku, K. Kida, Y. Fujiwara, K. Yamada, N. Yamamoto, S. Kobayashi, T. Saito, K. Wada, K. Satomi, K. Shinomiya, T. Tani, What Is the Best Multimodality Combination for Intraoperative Spinal Cord Monitoring of Motor Function? A Multicenter Study by the Monitoring Committee of the Japanese Society for Spine Surgery and Related Research, Global Spine J 6(3): 234-41, 2016. 
Figures \& Tables

Figure 1

CT scan that showed an example of OYL

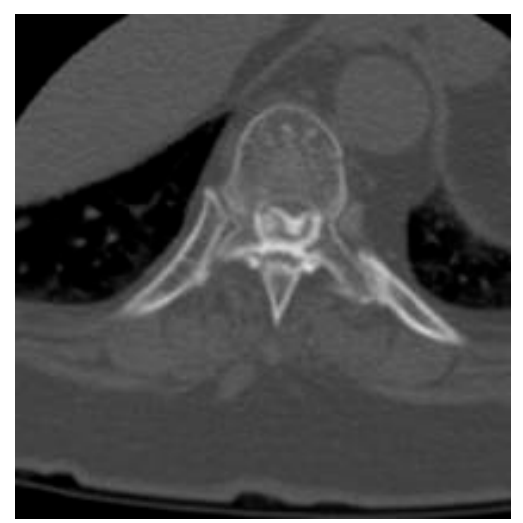


Figure 2: Frankel grade distribution in our series. Number of patients in each grade is highlighted by parenthesis. Majority were in Frankel grade D on presentation and most improved after operation.

Figure 2

\section{\begin{tabular}{|l|l|l|} 
Pre-op & Immediate post-op & 2 year post-op
\end{tabular}}

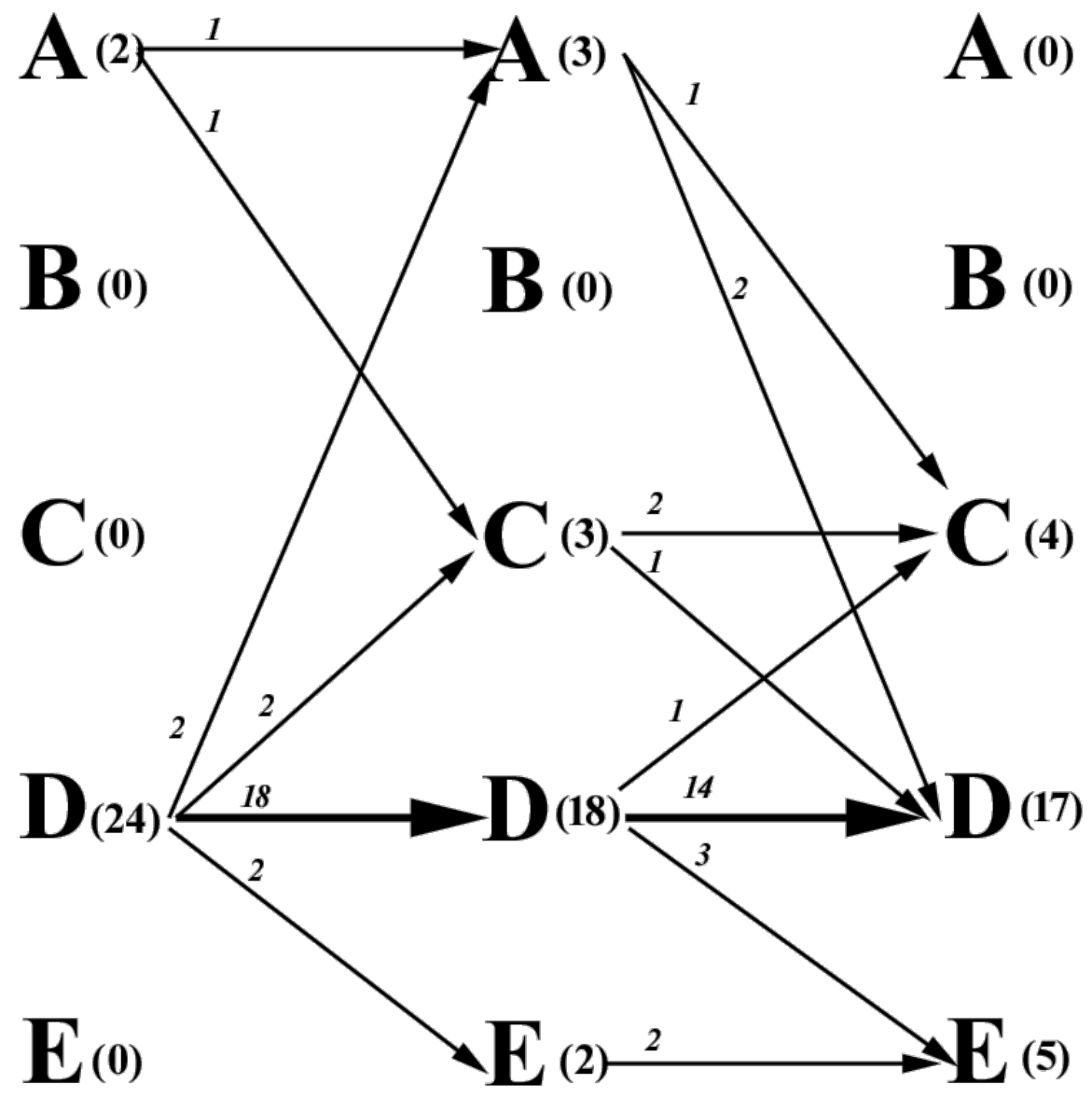


Table 1

Table 1: Frankel grade classification ${ }^{16}$

\begin{tabular}{|l|l|}
\hline Grade & Description \\
\hline A & Complete paralysis \\
\hline B & Sensory function only below injury level \\
\hline C & Incomplete motor function below injury \\
\hline D & level \\
\hline E & Fair to good motor function below injury \\
\hline
\end{tabular}


Table 2

Table 2: Summary of the neurological status

\begin{tabular}{|c|c|c|}
\hline Category & $\begin{array}{l}\text { Immediate } \\
\text { Postoperative (\%) }\end{array}$ & $\begin{array}{l}2 \text { year Postoperative } \\
(\%)\end{array}$ \\
\hline Motor improvement & 11 patients $(42.3 \%)$ & 14 patients (53.8\%) \\
\hline Sensory improvement & 19 patients $(73.1 \%)$ & 24 patients $(92.3 \%)$ \\
\hline Sphincter function & $\begin{array}{l}\text { The only } 1 \text { pati } \\
\text { disturbance had } \\
\text { immediately after of }\end{array}$ & $\begin{array}{l}\text { oresented with sphincter } \\
\text { on of sphincter function }\end{array}$ \\
\hline $\begin{array}{l}\text { Walking ability } \\
\text { improvement }\end{array}$ & 8 patients (30.8\%) & 16 patients $(61.5 \%)$ \\
\hline Neurological improvement & 21 patients $(80.8 \%)$ & 24 patients $(92.3 \%)$ \\
\hline Residual motor deficit & $\begin{array}{l}15 \text { patients }(57.7 \%) \\
\text { ( } 6 \text { less severe) }\end{array}$ & $\begin{array}{l}10 \text { patients }(38.5 \%) \\
\text { (4 less severe) }\end{array}$ \\
\hline Residual sensory deficit & $\begin{array}{l}23 \text { patients }(88.5 \%) \\
\text { (16 less severe) }\end{array}$ & $\begin{array}{l}14 \text { patients }(53.8 \%) \\
\text { (12 less severe) }\end{array}$ \\
\hline
\end{tabular}


Table 3

Table 3: Mean mJOA score changes at different levels of OYL

\begin{tabular}{|l|l|}
\hline Region of OYL & Mean JOA score change at 2-year follow up \\
\hline Upper thoracic & 1.43 \\
\hline Middle thoracic & 0.83 \\
\hline Lower thoracic & 1.60 \\
\hline P value & 0.71 \\
\hline
\end{tabular}

mJOA: modified Japanese Orthopaedic Association

OYL: ossified yellow ligament 
Table 4

Table 4: Intra-operative SSEP monitoring for spinal cord injury (22 out of 26)

\begin{tabular}{|l|l|l|l|}
\hline & $\begin{array}{l}\text { Unrecordable } \\
\text { pre-op }\end{array}$ & $\begin{array}{l}\text { Intra-op } \\
\text { significant drop }\end{array}$ & significant drop \\
\hline No neurological & 7 & 0 & 12 \\
\hline deterioration & & 1 & 1 \\
\hline Neurological & 1 & & \\
\hline deterioration & & & \\
\hline
\end{tabular}

